\title{
Smith and Rawls share a room
}

Citation for published version (APA):

Klaus, B. E., \& Klijn, F. (2007). Smith and Rawls share a room. METEOR, Maastricht University School of Business and Economics. METEOR Research Memorandum No. 026

https://doi.org/10.26481/umamet.2007026

Document status and date:

Published: 01/01/2007

DOI:

10.26481/umamet.2007026

Document Version:

Publisher's PDF, also known as Version of record

\section{Please check the document version of this publication:}

- A submitted manuscript is the version of the article upon submission and before peer-review. There can be important differences between the submitted version and the official published version of record.

People interested in the research are advised to contact the author for the final version of the publication, or visit the DOI to the publisher's website.

- The final author version and the galley proof are versions of the publication after peer review.

- The final published version features the final layout of the paper including the volume, issue and page numbers.

Link to publication

\footnotetext{
General rights rights.

- You may freely distribute the URL identifying the publication in the public portal. please follow below link for the End User Agreement:

www.umlib.nl/taverne-license

Take down policy

If you believe that this document breaches copyright please contact us at:

repository@maastrichtuniversity.nl

providing details and we will investigate your claim.
}

Copyright and moral rights for the publications made accessible in the public portal are retained by the authors and/or other copyright owners and it is a condition of accessing publications that users recognise and abide by the legal requirements associated with these

- Users may download and print one copy of any publication from the public portal for the purpose of private study or research.

- You may not further distribute the material or use it for any profit-making activity or commercial gain

If the publication is distributed under the terms of Article $25 \mathrm{fa}$ of the Dutch Copyright Act, indicated by the "Taverne" license above, 
Bettina Klaus, Flip Klijn

Smith and Rawls Share a Room

$\mathrm{RM} / 07 / 026$

JEL code: C62, C78

\section{METE@R}

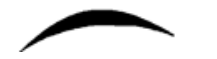

Maastricht research school of Economics

of TEchnology and ORganizations

Universiteit Maastricht

Faculty of Economics and Business Administration P.O. Box 616

NL - 6200 MD Maastricht

phone : ++31433883830

fax $\quad$ : ++31433884873 



\title{
Smith and Rawls Share a Room*
}

\author{
Bettina Klaus ${ }^{\dagger} \quad$ Flip Klijn
}

June 2007

\begin{abstract}
We consider one-to-one matching (roommate) problems in which agents (students) can either be matched as pairs or remain single. The aim of this paper is twofold. First, we review a key result for roommate problems (the "lonely wolf" theorem) for which we provide a concise and elementary proof. Second, and related to the title of this paper, we show how the often incompatible concepts of stability (represented by the political economist Adam Smith) and fairness (represented by the political philosopher John Rawls) can be reconciled for roommate problems.
\end{abstract}

JEL classification: C62, C78.

Keywords: roommate problem, stability, fairness.

\section{Roommate Markets}

We consider one-to-one matching problems in which agents can either be matched as pairs or remain single. Gale and Shapley (1962, Example 3) introduced these so-called roommate problems as follows: "An even number of boys wish to divide up into pairs of roommates." A very common extension of this problem is to allow also for odd numbers of agents and to consider the formation of pairs and singletons (rooms can be occupied either by one or by two agents). The class of roommate problems also include as special cases the well-known marriage problems (Gale and Shapley, 1962). ${ }^{1}$

*B. Klaus thanks the Netherlands Organisation for Scientific Research (NWO) for its support under grant VIDI-452-06-013. F. Klijn's research was supported through the Spanish Plan Nacional I+D+I (SEJ200501690) and the Generalitat de Catalunya (SGR2005-00626 and the Barcelona Economics Program of XREA).

${ }^{\dagger}$ Department of Economics, Maastricht University, P.O. Box 616, 6200 MD Maastricht, The Netherlands; e-mail: b.klaus@algec.unimaas.nl

${ }^{\ddagger}$ Corresponding author: Institute for Economic Analysis (CSIC), Campus UAB, 08193 Bellaterra (Barcelona), Spain; e-mail: flip.klijn@iae.csic.es

${ }^{1}$ There is a large literature on the marriage problem; see, for instance, Roth and Sotomayor (1990) and the two-sided matching bibliography on Al Roth's game theory, experimental economics, and market design page. In comparison, relatively few papers and books deal with roommate problems; some of the key references concerning roommate problems are Chung (2000); Diamantoudi et al. (2004); Gusfield and Irving (1989); Tan (1991). 
The aim of this paper is twofold. First, we review a key result for roommate problems for which we provide a concise and elementary proof. Second, and related to the title of this paper, we show how the often incompatible concepts of equilibrium or stability (represented by Adam Smith ${ }^{2}$ ) and fairness (represented by John Rawls ${ }^{3}$ ) can be reconciled for roommate problems.

Formally, a roommate problem (Gale and Shapley, 1962) is a pair $\left(N,\left(\succeq_{i}\right)_{i \in N}\right)$ where $N$ is a finite set of agents and, for each $i \in N, \succeq_{i}$ is a total order over $N{ }^{4}$ For each $i \in N$, we interpret $\succeq_{i}$ as agent $i$ 's preferences over sharing a room with any of the agents in $N \backslash\{i\}$ and having a room for himself (or consuming an outside option such as living off-campus). Preferences are strict, i.e., $k \succeq_{i} j$ and $j \succeq_{i} k$ if and only if $j=k$. The strict preference relation associated with $\succeq_{i}$ is denoted by $\succ_{i}$. A solution to a roommate problem, a matching $\mu$, is a partition of $N$ in pairs and singletons. Alternatively, we describe a matching by a function $\mu: N \rightarrow N$ of order two, i.e., for all $i \in N, \mu(\mu(i))=i$. Agent $\mu(i)$ is agent $i$ 's match, i.e., the agent with whom he is matched to share a room (possibly himself). If $\mu(i)=i$ then we call $i$ a single.

A marriage problem (Gale and Shapley, 1962) is a roommate problem $\left(N,\left(\succeq_{i}\right)_{i \in N}\right)$ such that $N$ is the union of two disjoint sets $M$ and $W$ (men and women), and each agent in $M$ (respectively $W$ ) prefers being alone to being matched with any other agent in $M$ (respectively $W$ ).

A matching $\mu$ is blocked by a pair $\{i, j\} \subseteq N$ (possibly $i=j$ ) if $j \succ_{i} \mu(i)$ and $i \succ_{j} \mu(j)$. If $\{i, j\}$ blocks $\mu$, then $\{i, j\}$ is called a blocking pair for $\mu$. A matching is individually rational if there is no blocking pair $\{i, j\}$ with $i=j$. A matching is stable if there is no blocking pair. A roommate problem is solvable if the set of stable matchings is non-empty. Gale and Shapley (1962) showed that all marriage problems are solvable and provided an unsolvable roommate problem (Gale and Shapley, 1962, Example 3).

The following is a simplified version of Gale and Shapley's example with three agents: $2 \succ_{1} 3 \succ_{1} 1,3 \succ_{2} 1 \succ_{2} 2$, and $1 \succ_{3} 2 \succ_{3} 3$. Clearly, the matching where all agents are singles is not stable (any two agents can block). So, assume two agents share a room. Then, the single agent is the best roommate for one of these two agents and hence a blocking pair can be formed. Tan (1991) provided a necessary and sufficient condition for the existence of a stable matching.

Note that for marriage problems, an individually rational matching never matches two men or two women, i.e., the partition consists of man-woman pairs and singletons.

\section{Lonely Wolves, Medians, and Compromise}

Our starting point is a solvable roommate problem. Typically there are multiple stable matchings and with choice comes the opportunity to select a particularly appealing stable

\footnotetext{
${ }^{2}$ Adam Smith (1723-1790), a political economist, propagated the view that individuals even though interested only in their own gains will still advance public interest (Smith, 1796).

${ }^{3}$ John Rawls (1921-2002), a political philosopher, discussed important aspects of fairness and justice particularly suited for economic applications (Rawls, 1971).

${ }^{4} \mathrm{~A}$ total order is a binary relation that satisfies reflexivity, antisymmetry, transitivity, and totality or comparability.
} 
matching, for example a stable matching that maximizes the number of matched pairs. It turns out that no such selection is possible because an agent who is single at one stable matching is also single at all other stable matchings (Gusfield and Irving, 1989, Theorem 4.5.2). In fact, Gale and Sotomayor (1985, Proposition 1) already proved this "lonely wolf" theorem for marriage problems. Here we provide an elementary proof.

\section{Theorem 1 The lonely wolf theorem}

Let $\mu$ and $\mu^{\prime}$ be stable matchings. Then, $\mu$ and $\mu^{\prime}$ have the same set of single agents, i.e., $\mu(i)=i \Leftrightarrow \mu^{\prime}(i)=i$.

Proof Suppose $\mu\left(i_{1}\right)=i_{1} \neq i_{2}=\mu^{\prime}\left(i_{1}\right)$ for some $i_{1}, i_{2} \in N$. Since preferences are strict and $\mu^{\prime}$ is individually rational, $i_{2} \succ_{i_{1}} i_{1}$.

Define $i_{3}:=\mu\left(i_{2}\right)$. Then, $i_{3} \succ_{i_{2}} i_{1}$ (otherwise $\left\{i_{1}, i_{2}\right\}$ is a blocking pair for $\mu$ ) and $i_{3} \neq i_{2}$ (otherwise $\left\{i_{2}\right\}$ is a blocking pair for $\mu^{\prime}$ ). Define $i_{4}:=\mu^{\prime}\left(i_{3}\right)$. Then, $i_{4} \succ_{i_{3}} i_{2}$ (otherwise $\left\{i_{2}, i_{3}\right\}$ is a blocking pair for $\mu^{\prime}$ ) and $i_{4} \neq i_{3}$ (otherwise $\left\{i_{3}\right\}$ is a blocking pair for $\mu$ ). Next, define agents $i_{5}, i_{6}, \ldots$ by

$$
i_{p+1}:= \begin{cases}\mu\left(i_{p}\right) & \text { if } p \text { is even; } \\ \mu^{\prime}\left(i_{p}\right) & \text { if } p \text { is odd }\end{cases}
$$

Similarly as before it follows that for all $p \geq 2, i_{p+1} \succ_{i_{p}} i_{p-1}$ and $i_{p+1} \neq i_{p}$. Since $N$ is finite, there is a $p^{\prime} \geq 1$ with

$$
i_{p^{\prime}+1}=i_{l} \text { for some } l \leq p^{\prime} .
$$

Let $\bar{p}$ be the smallest $p^{\prime} \geq 1$ that satisfies (1). By showing that this leads to a contradiction, we prove that $\mu\left(i_{1}\right)=i_{1}$ implies $\mu^{\prime}\left(i_{1}\right)=i_{1}$.

Note that $i_{3} \notin\left\{i_{1}, i_{2}\right\}$ and therefore $\bar{p} \geq 3$. In general, for all $p \geq 2, i_{p+1} \notin\left\{i_{p}, i_{p-1}\right\}$. Hence, $\bar{p} \geq 3$ and $\bar{p}>l+1$ for all $l \leq \bar{p}$ with $i_{\bar{p}+1}=i_{l}$.

Let $l \leq \bar{p}$ with $i_{\bar{p}+1}=i_{l}$. Suppose $\bar{p}$ is even, i.e., $i_{\bar{p}+1}=\mu\left(i_{\bar{p}}\right)$. Then, $\mu\left(i_{\bar{p}}\right)=i_{l}$. If $l=1$, then from the definition of $i_{1}, i_{\bar{p}}=i_{1}$, contradicting that $\bar{p}$ is the smallest $p^{\prime} \geq 1$ that satisfies (1). If $l$ is odd and $l \neq 1$, then $i_{l-1}=\mu\left(i_{l}\right)=i_{\bar{p}}$, contradicting the definition of $\bar{p}$. If $l$ is even, then $i_{l+1}=\mu\left(i_{l}\right)=i_{\bar{p}}$, contradicting the definition of $\bar{p}$.

Suppose $\bar{p}$ is odd, i.e., $i_{\bar{p}+1}=\mu^{\prime}\left(i_{\bar{p}}\right)$. Then, $\mu^{\prime}\left(i_{\bar{p}}\right)=i_{l}$. If $l=1$, then from the definition of $i_{2}, i_{\bar{p}}=i_{2}$, contradicting the definition of $\bar{p}$. If $l$ is odd and $l \neq 1$, then $i_{l+1}=\mu^{\prime}\left(i_{l}\right)=i_{\bar{p}}$, contradicting the definition of $\bar{p}$. If $l$ is even, then $i_{l-1}=\mu^{\prime}\left(i_{l}\right)=i_{\bar{p}}$, contradicting the definition of $\bar{p}$.

Since no selection can be based on the set of matched agent, we next try to find a stable matching that will be perceived as fair by the agents. Imagine that we ask each agent to rank all stable matchings according to his preferences. For two (stable) matchings $\mu$ and $\mu^{\prime}$, $\mu \succeq_{i} \mu^{\prime} \Leftrightarrow \mu(i) \succeq_{i} \mu^{\prime}(i)$. Note that since an agent might be matched to the same agent in several stable matchings, this ranking is not strict. Clearly, we cannot always give the best match to every agent, but can we implement fairness by finding a matching that matches each agent with his $k$-th ranked match? We explain this idea in our next example and show that this idea of fairness or compromise (at least if there is an even number of stable matchings) is not always feasible. 


\section{Example 1 An even number of stable matchings and no compromise}

Consider the following roommate problem with 8 agents and preferences as listed in the table below. The first column, for instance, means that $8 \succ_{1} 2 \succ_{1} 7 \succ_{1} 4 \succ_{1} 1 \succ_{1} 5 \succ_{1} 3 \succ_{1} 6$.

\begin{tabular}{cccccccc}
\hline$\succ_{1}$ & $\succ_{2}$ & $\succ_{3}$ & $\succ_{4}$ & $\succ_{5}$ & $\succ_{6}$ & $\succ_{7}$ & $\succ_{8}$ \\
\hline \hline 8 & 3 & 4 & 2 & 6 & 7 & 8 & 5 \\
2 & 5 & 6 & 1 & 3 & 5 & 6 & 7 \\
7 & 1 & 2 & 3 & 8 & 4 & 1 & 1 \\
4 & 4 & 5 & 5 & 2 & 2 & 2 & 2 \\
1 & 6 & 1 & 6 & 1 & 6 & 3 & 8 \\
5 & 8 & 7 & 8 & 5 & 1 & 4 & 6 \\
3 & 7 & 3 & 7 & 7 & 3 & 5 & 4 \\
6 & 2 & 8 & 4 & 4 & 8 & 7 & 3 \\
\hline
\end{tabular}

There are 4 stable matchings:

$$
\begin{aligned}
& \mu_{1}=\{\{1,2\},\{3,4\},\{5,6\},\{7,8\}\} \\
& \mu_{2}=\{\{1,2\},\{3,4\},\{5,8\},\{6,7\}\} \\
& \mu_{3}=\{\{1,4\},\{2,3\},\{5,6\},\{7,8\}\} \\
& \mu_{4}=\{\{1,4\},\{2,3\},\{5,8\},\{6,7\}\}
\end{aligned}
$$

The following (weak) rankings on matchings are induced ( $\sim$ denotes indifferences):

$$
\begin{aligned}
& \mu_{1} \sim_{1} \mu_{2} \succ_{1} \mu_{3} \sim_{1} \mu_{4} \\
& \mu_{3} \sim_{2} \mu_{4} \succ_{2} \mu_{1} \sim_{2} \mu_{2} \\
& \mu_{1} \sim_{3} \mu_{2} \succ_{3} \mu_{3} \sim_{3} \mu_{4} \\
& \mu_{3} \sim_{4} \mu_{4} \succ_{4} \mu_{1} \sim_{4} \mu_{2} \\
& \mu_{1} \sim_{5} \mu_{3} \succ_{5} \mu_{2} \sim_{5} \mu_{4} \\
& \mu_{2} \sim_{6} \mu_{4} \succ_{6} \mu_{1} \sim_{6} \mu_{3} \\
& \mu_{1} \sim_{7} \mu_{3} \succ_{7} \mu_{2} \sim_{7} \mu_{4} \\
& \mu_{2} \sim_{8} \mu_{4} \succ_{8} \mu_{1} \sim_{8} \mu_{3}
\end{aligned}
$$

Agents 1, 2, and 4 order their matches at the 4 stable matchings as 2, 2, 4, 4; 3, 3, 1, 1; and $1,1,3,3$ respectively. If agent 1 is given his first or second choice, then he is matched with agent 2 who then receives his third or fourth choice. If agent 1 is given his third or fourth choice, then he is matched with agent 4 who then receives his first or second choice. It follows that matching all agents with a $k$-th ranked match is not possible.

The impossibility result exhibited in the example is due to the fact that there is an even number of (distinct) stable matchings. Next, we show that for roommate problems with an odd number of stable matchings a compromise matching where each agent is matched to a match of the same rank is possible. In fact, we prove that for any odd number of stable matchings, a stable matching at which each agent is matched to his "median" match always exists. Thus, for roommate problems with an odd number of stable matchings Adam Smith (who stands for stability) and John Rawls (who stands for fairness) represent compatible criteria and hence "can share a room." 5

\footnotetext{
${ }^{5}$ Example 1 is an example where the equilibrium and the fairness principles represented by Adam Smith and John Rawls are incompatible: there they will never be able to share a room.
} 
The next lemma, which appeared in Gusfield and Irving (1989, Lemma 4.3.9), facilitates the proof of our main result.

Lemma 1 Let $\mu$ and $\mu^{\prime}$ be stable matchings. Let $i \in N$. Suppose $\mu^{\prime}(i) \neq \mu(i)=j$ for some $j \in N$. Then, $j, \mu^{\prime}(i) \neq i$. Moreover, $\mu(i) \succ_{i} \mu^{\prime}(i)$ implies $\mu^{\prime}(j) \succ_{j} \mu(j)$.

Proof By Theorem 1, j, $\mu^{\prime}(i) \neq i$. Suppose $\mu(i) \succ_{i} \mu^{\prime}(i)$ and $\mu(j) \succeq_{j} \mu^{\prime}(j)$. Since preferences are strict and $\mu(j)=i \neq \mu^{\prime}(j), \mu(j) \succ_{j} \mu^{\prime}(j)$. Hence, $\{i, j\}$ is a blocking pair for $\mu^{\prime}$, contradicting stability.

Our main result, Theorem 2, extends Theorem 4.3.5 in Gusfield and Irving (1989) from three to any odd number of stable matchings.

Let $\mu_{1}, \ldots, \mu_{2 k+1}$ be an odd number of (possibly non-distinct) stable matchings. Let each agent rank these matchings according to his preferences as explained before (Example 1). We denote agent $i$ 's $(k+1)$-st ranked match by $\operatorname{med}\left\{\mu_{1}(i), \ldots, \mu_{2 k+1}(i)\right\}$.

\section{Theorem 2 Smith and Rawls share a room}

Let $\mu_{1}, \ldots, \mu_{2 k+1}$ be an odd number of (possibly non-distinct) stable matchings. Then, $\mu^{*}$ : $N \rightarrow N$ defined by

$$
\mu^{*}(i):=\operatorname{med}\left\{\mu_{1}(i), \ldots, \mu_{2 k+1}(i)\right\} \text { for all } i \in N
$$

is a well-defined stable matching. We call $\mu^{*}$ the median matching of $\mu_{1}, \ldots, \mu_{2 k+1}$.

In the context of the linear programming approach to so-called bistable matching problems, Sethuraman and Teo (2001, Theorem 3.2) mentioned this result (without proof) as an interesting structural property of stable roommate matchings. Here we provide an elementary proof.

Proof First, we show that $\mu^{*}$ is a well-defined matching, i.e., $\mu^{*}$ is of order 2. Let $i \in N$ with $\mu^{*}(i) \neq i$. We have to prove that $\mu^{*}\left(\mu^{*}(i)\right)=i$. Let $j:=\mu^{*}(i)$. Without loss of generality, $\mu_{1}(i) \succeq_{i} \mu_{2}(i) \succeq_{i} \cdots \succeq_{i} \mu_{2 k}(i) \succeq_{i} \mu_{2 k+1}(i)$. Then, $\mu^{*}(i)=\mu_{k+1}(i)=j$ and $\mu_{k+1}(j)=i$. Let $S=\left\{\mu_{1}, \ldots, \mu_{2 k+1}\right\}$. Define

$$
\begin{aligned}
\bar{B}_{i} & :=\left\{\mu \in S \mid \mu \succeq_{i} \mu_{k+1}\right\}, \\
W_{i} & :=\left\{\mu \in S \mid \mu \prec_{i} \mu_{k+1}\right\}, \\
B_{j} & :=\left\{\mu \in S \mid \mu \succ_{j} \mu_{k+1}\right\}, \text { and } \\
\bar{W}_{j} & :=\left\{\mu \in S \mid \mu \preceq_{j} \mu_{k+1}\right\} .
\end{aligned}
$$

Clearly, $\left\{\bar{B}_{i}, W_{i}\right\}$ and $\left\{B_{j}, \bar{W}_{j}\right\}$ are partitions of $S$, where possibly $W_{i}=\emptyset$ or $B_{j}=\emptyset$.

By Lemma $1, W_{i} \subseteq B_{j}$. Suppose there exists $\mu \in B_{j} \backslash W_{i}$. Since $\bar{B}_{i} \cup W_{i}=S, \mu \in \bar{B}_{i}$. Thus, $\mu \in \bar{B}_{i} \cap B_{j}$. Since $\mu \in B_{j}, \mu \succ_{j} \mu_{k+1}$. In particular, since $\mu_{k+1}(j)=i, \mu(j) \neq i$. Then, since $\mu \in \bar{B}_{i}, \mu \succ_{i} \mu_{k+1}$. Hence, $\{i, j\}$ is a blocking pair for $\mu_{k+1}$, contradicting stability. So, $W_{i}=B_{j}$. Since $\left\{\bar{B}_{i}, W_{i}\right\}$ and $\left\{B_{j}, \bar{W}_{j}\right\}$ are partitions of $S, \bar{B}_{i}=\bar{W}_{j}$. Hence,

$$
\begin{aligned}
\overbrace{\mu_{1} \succeq_{i} \mu_{2} \succeq_{i} \cdots \succeq_{i} \mu_{k+1} \sim_{i} \cdots}^{\bar{B}_{i}} & \succ_{i} \\
W_{i} & \succ_{j} \underbrace{\cdots \sim_{j} \mu_{k+1} \succeq_{j}\left\{\mu_{1}, \ldots, \mu_{k}\right\}}_{\bar{B}_{i}}
\end{aligned}
$$


In particular, it follows that $\mu^{*}(j)=\mu_{k+1}(j)$. Hence, $\mu^{*}\left(\mu^{*}(i)\right)=\mu^{*}(j)=\mu_{k+1}(j)=i$.

We now prove that $\mu^{*}$ is stable. By definition, $\mu^{*}$ is individually rational. Suppose there is a blocking pair $\{i, j\}$ with $i \neq j$, i.e., $j \succ_{i} \mu^{*}(i)$ and $i \succ_{j} \mu^{*}(j)$. Then, $i$ prefers $j$ to at least $k+1$ stable matchings in $\left\{\mu_{1}, \ldots, \mu_{2 k+1}\right\}$. Similarly, $j$ prefers $i$ to at least $k+1$ stable matchings in $\left\{\mu_{1}, \ldots, \mu_{2 k+1}\right\}$. Hence, for at least one stable matching $\mu \in\left\{\mu_{1}, \ldots, \mu_{2 k+1}\right\}$, $j \succ_{i} \mu(i)$ and $i \succ_{j} \mu(j)$, contradicting stability.

We can easily extend the result of Theorem 2 to an even number of stable matchings (Sethuraman and Teo, 2001, Theorem 3.3).

\section{Corollary 1 Smith and Rawls (almost) share a room}

Let $\mu_{1}, \ldots, \mu_{2 k}$ be an even number of (possibly non-distinct) stable matchings. Then, there exists a stable matching at which each agent is assigned a match of rank $k$ or $k+1$.

Proof By Theorem 2, the median of the first $2 k-1$ stable matchings, i.e., $\mu^{*}=$ $\operatorname{med}\left\{\mu_{1}, \ldots, \mu_{2 k-1}\right\}$, is a well-defined and stable matching. Then, for any $i \in N$,

agent $i$ is matched at $\mu^{*}$ with his $\begin{cases}k \text {-th ranked match } & \text { if } \mu^{*}(i) \succeq_{i} \mu_{2 k}(i) ; \\ (k+1) \text {-st ranked match } & \text { if } \mu^{*}(i) \preceq_{i} \mu_{2 k}(i) .\end{cases}$

In the next example we calculate the median matching for three stable matchings and demonstrate that the "median operator" is not closed, i.e., that the resulting median matching is not always one of the stable matchings that were used to calculate it.

\section{Example 2 An odd number of stable matchings and median matchings}

Consider the following roommate problem with 8 agents and preferences as listed in the table below.

\begin{tabular}{cccccccc}
\hline$\succ_{1}$ & $\succ_{2}$ & $\succ_{3}$ & $\succ_{4}$ & $\succ_{5}$ & $\succ_{6}$ & $\succ_{7}$ & $\succ_{8}$ \\
\hline \hline 4 & 7 & 2 & 6 & 2 & 1 & 4 & 7 \\
2 & 4 & 8 & 2 & 6 & 2 & 6 & 2 \\
5 & 6 & 5 & 5 & 4 & 8 & 1 & 5 \\
3 & 8 & 4 & 8 & 7 & 3 & 3 & 1 \\
8 & 1 & 6 & 3 & 8 & 4 & 5 & 4 \\
7 & 5 & 1 & 1 & 3 & 5 & 2 & 6 \\
6 & 3 & 7 & 7 & 1 & 7 & 8 & 3 \\
1 & 2 & 3 & 4 & 5 & 6 & 7 & 8 \\
\hline
\end{tabular}

There are 5 stable matchings:

$$
\begin{aligned}
& \mu_{1}=\{\{1,3\},\{2,4\},\{5,7\},\{6,8\}\} \\
& \mu_{2}=\{\{1,3\},\{2,7\},\{4,5\},\{6,8\}\} \\
& \mu_{3}=\{\{1,7\},\{2,4\},\{3,6\},\{5,8\}\} \\
& \mu_{4}=\{\{1,8\},\{2,4\},\{3,6\},\{5,7\}\} \\
& \mu_{5}=\{\{1,8\},\{2,7\},\{3,6\},\{4,5\}\}
\end{aligned}
$$

$\mu_{4}$ is the median matching of $\left\{\mu_{1}, \mu_{2}, \mu_{3}, \mu_{4}, \mu_{5}\right\}$ and of $\left\{\mu_{1}, \mu_{3}, \mu_{5}\right\}$. 
For marriage problems we even have a stronger result. Let $\mu_{1}, \ldots, \mu_{k}$ be $k$ (possibly nondistinct) stable matchings for a marriage problem and assume that each agent ranks these matchings according to his/her preferences. Using linear programming tools, Teo and Sethuraman (1998, Theorem 2) showed that the map that assigns to each man his $l$-th (weakly) best match and to each woman her $(k-l+1)$-st (weakly) best match determines a welldefined and stable matching. We explain and discuss (generalized) medians as compromise solutions for two-sided matching problems in Klaus and Klijn (2006). Below we provide an elementary proof of this "generalized median result" for marriage problems.

Consider a marriage problem $\left(M \cup W,\left(\succeq_{i}\right)_{i \in N}\right)$. Let $\mu_{1}, \ldots, \mu_{k}$ be $k$ (possibly non-distinct) stable matchings. Let each agent rank these matchings according to his/her preferences as explained before. Formally, for each $i \in M \cup W$ there is a sequence of matchings $\left(\mu_{1}^{i}, \ldots, \mu_{k}^{i}\right)$ such that $\left\{\mu_{1}^{i}, \ldots, \mu_{k}^{i}\right\}=\left\{\mu_{1}, \ldots, \mu_{k}\right\}$ and for any $l \in\{1, \ldots, k-1\}, \mu_{l}^{i}(i) \succeq_{i} \mu_{l+1}^{i}(i)$. Thus, for any $l \in\{1, \ldots, k\}$, at $\mu_{l}^{i}$ agent $i$ is assigned to his/her $l$-th (weakly) best match (among the $k$ stable matchings).

For any $l \in\{1, \ldots, k\}$, we define the generalized median stable matching $\alpha_{l}$ as the function $\alpha_{l}: M \cup W \rightarrow M \cup W$ defined by

$$
\alpha_{l}(i):= \begin{cases}\mu_{l}^{i}(i) & \text { if } i \in M \\ \mu_{(k-l+1)}^{i}(i) & \text { if } i \in W\end{cases}
$$

\section{Theorem 3 Marriage and compromise (generalized medians)}

Let $\mu_{1}, \ldots, \mu_{k}$ be $k$ (possibly non-distinct) stable matchings for a marriage problem. Then, for any $l \in\{1, \ldots, k\}, \alpha_{l}$ is a well-defined stable matching.

Proof Let $l \in\{1, \ldots, k\}$. First, we show that $\alpha_{l}$ is a well-defined matching, i.e., $\alpha_{l}$ is of order 2. Let $m \in M$ with $\alpha_{l}(m)=w \in W$. We have to prove that $\alpha_{l}\left(\alpha_{l}(m)\right)=m$. Without loss of generality, $\mu_{1}(m) \succeq_{m} \mu_{2}(m) \succeq_{m} \cdots \succeq_{m} \mu_{l}(m) \succeq_{m} \cdots \succeq_{m} \mu_{k-1}(m) \succeq_{m} \mu_{k}(m)$ and $\alpha_{l}(m)=\mu_{l}(m)$. Then, $\mu_{l}(m)=\alpha_{l}(m)=w$ and $\mu_{l}(w)=m$. By arguments similar to those in the first part of the proof of Theorem $2,{ }^{6}$

$$
\begin{aligned}
\mu_{1} \succeq_{m} \cdots \succeq_{m} \mu_{l-1} \succeq_{m} \quad \mu_{l} \succeq_{m} \mu_{l+1} \succeq_{m} \cdots \succeq_{m} \mu_{k} \\
\left\{\mu_{k}, \ldots, \mu_{l+1}\right\} \succeq_{w} \quad \mu_{l} \quad \succeq_{w}\left\{\mu_{l-1}, \ldots, \mu_{1}\right\}
\end{aligned}
$$

In particular, $\mu_{l}(w)$ is the $(k-l+1)$-st ranked match for woman $w$ and therefore $\alpha_{l}(w)=$ $\mu_{l}(w)$. Hence, $\alpha_{l}\left(\alpha_{l}(m)\right)=\alpha_{l}(w)=\mu_{l}(w)=m$.

We now prove that $\alpha_{l}$ is stable. By definition, $\alpha_{l}$ is individually rational. Suppose there is a blocking pair $\{m, w\}$ with $m \in M$ and $w \in W$, i.e., $w \succ_{m} \alpha_{l}(m)$ and $m \succ_{w} \alpha_{l}(w)$. Then, $m$ prefers $w$ to at least $k-l+1$ stable matchings in $\left\{\mu_{1}, \ldots, \mu_{k}\right\}$. Similarly, $w$ prefers $m$ to at least $l$ stable matchings in $\left\{\mu_{1}, \ldots, \mu_{k}\right\}$. Hence, for at least one matching $\mu \in\left\{\mu_{1}, \ldots, \mu_{k}\right\}$, $w \succ_{m} \mu(m)$ and $m \succ_{w} \mu(w)$, contradicting stability.

\footnotetext{
${ }^{6}$ Recall that in the first part of the proof of Theorem 2 only the relative rankings of agent $i$ 's mates with respect to $\mu_{k+1}$ mattered - which exact rank $\mu_{k+1}$ had did not play any role in that part of the proof.
} 
Fleiner (2002, Theorem 5.5), Klaus and Klijn (2006, Theorem 3.2), and Sethuraman et al. (2006, Theorem 9) generalized Theorem 3 to college admissions problems (Gale and Shapley, 1962) with responsive preferences, ${ }^{7}$ in which students have to be matched to colleges based on the students' and the colleges' preferences over the other side of the market and colleges' capacity constraints. It is well-known that for this class of two-sided matching problems the set of stable matchings is still non-empty and has the "lonely wolf" property, i.e., the set of single students does not vary from one stable matching to another (see, for instance, Roth and Sotomayor, 1990, Lemma 5.6 and Theorem 5.12). Martínez et al. (2000) introduced the domain of $q$-separable and substitutable preferences for colleges that contains the domain of responsive preferences. They prove that even for this larger domain the set of stable matchings is non-empty and that the "lonely wolf" property holds. Surprisingly, we cannot extend Theorem 3 to the domain of $q$-separable and substitutable preferences as the following example shows.

\section{Example 3 No compromise for q-separable and substitutable preferences}

Consider Martínez et al.'s (2000, Example 2) college admissions problem with 4 students $s_{1}, s_{2}, s_{3}, s_{4}, 2$ colleges $C_{1}$ and $C_{2}$ with 2 seats each, and preferences as listed in the table below. The colleges' preferences are $q$-separable and substitutable (for details see Martínez et al., 2000).

\begin{tabular}{cccccc}
\hline$\succ_{C_{1}}$ & $\succ_{C_{2}}$ & $\succ_{s_{1}}$ & $\succ_{s_{2}}$ & $\succ_{s_{3}}$ & $\succ_{s_{4}}$ \\
\hline \hline$\left\{s_{1}, s_{2}\right\}$ & $\left\{s_{3}, s_{4}\right\}$ & $C_{2}$ & $C_{2}$ & $C_{1}$ & $C_{1}$ \\
$\left\{s_{1}, s_{3}\right\}$ & $\left\{s_{2}, s_{4}\right\}$ & $C_{1}$ & $C_{1}$ & $C_{2}$ & $C_{2}$ \\
$\left\{s_{2}, s_{4}\right\}$ & $\left\{s_{1}, s_{3}\right\}$ & & & & \\
$\left\{s_{3}, s_{4}\right\}$ & $\left\{s_{1}, s_{2}\right\}$ & & & & \\
$\left\{s_{1}, s_{4}\right\}$ & $\left\{s_{1}, s_{4}\right\}$ & & & & \\
$\left\{s_{2}, s_{3}\right\}$ & $\left\{s_{2}, s_{3}\right\}$ & & & & \\
$\left\{s_{1}\right\}$ & $\left\{s_{1}\right\}$ & & & & \\
$\left\{s_{2}\right\}$ & $\left\{s_{2}\right\}$ & & & & \\
$\left\{s_{3}\right\}$ & $\left\{s_{3}\right\}$ & & & & \\
$\left\{s_{4}\right\}$ & $\left\{s_{4}\right\}$ & & & & \\
\hline
\end{tabular}

There are 4 stable matchings:

$$
\begin{aligned}
& \mu_{1}=\left\{\left\{C_{1}, s_{1}, s_{2}\right\},\left\{C_{2}, s_{3}, s_{4}\right\}\right\} \\
& \mu_{2}=\left\{\left\{C_{1}, s_{1}, s_{3}\right\},\left\{C_{2}, s_{2}, s_{4}\right\}\right\} \\
& \mu_{3}=\left\{\left\{C_{1}, s_{2}, s_{4}\right\},\left\{C_{2}, s_{1}, s_{3}\right\}\right\} \\
& \mu_{4}=\left\{\left\{C_{1}, s_{3}, s_{4}\right\},\left\{C_{2}, s_{1}, s_{2}\right\}\right\}
\end{aligned}
$$

Considering the first three matchings, one straightforwardly checks that matching each agent with its median match is not a matching: $C_{1}$ would be matched with $\left\{s_{1}, s_{3}\right\}$ but at the same time $s_{3}$ would be matched with $C_{2}$.

\footnotetext{
${ }^{7}$ By responsiveness (Roth, 1985), a college's preference relation over sets of students is related to its ranking of single students in the following way: the college always prefers to add an acceptable student to any set of students (provided this does not violate the capacity constraint) and it prefers to replace any student by a better student.
} 


\section{References}

Chung, K.-S. (2000): "On the Existence of Stable Roommate Matchings." Games and Economic Behavior, 33: 206-230.

Diamantoudi, E., Miyagawa, E., and Xue, L. (2004): "Random Paths to Stability in the Roommate Problem." Games and Economic Behavior, 48: 18-28.

Fleiner, T. (2002): "Some Results on Stable Matchings and Fixed Points." Technical Report TR-2002-08, Egerváry Research Group, Budapest. http://www.cs.elte.hu/egres.

Gale, D. and Shapley, L. S. (1962): "College Admissions and the Stability of Marriage." American Mathematical Monthly, 69: 9-15.

Gale, D. and Sotomayor, M. A. O. (1985): "Ms. Machiavelli and the Stable Matching Problem." American Mathematical Monthly, 92: 261-268.

Gusfield, D. and Irving, R. W. (1989): The Stable Marriage Problem: Structure and Algorithms. The MIT Press, Cambridge.

Klaus, B. and Klijn, F. (2006): "Median Stable Matching for College Admissions." International Journal of Game Theory, 34: 1-11.

Martínez, R., Massó, J., Neme, A., and Oviedo, J. (2000): "Single Agents and the Set of Many-to-One Stable Matchings." Journal of Economic Theory, 91: 91-105.

Rawls, J. (1971): A Theory of Justice. Harvard University Press, Cambridge.

Roth, A. E. (1985): "The College Admissions Problem is not Equivalent to the Marriage Problem." Journal of Economic Theory, 36: 277-288.

Roth, A. E. and Sotomayor, M. A. O. (1990): Two-Sided Matching: A Study in GameTheoretic Modeling and Analysis. Cambridge University Press, Cambridge.

Sethuraman, J. and Teo, C.-P. (2001): "A Polynomial-Time Algorithm for the Bistable Roommates Problem." Journal of Computer and Systems Sciences, 63: 486-497.

Sethuraman, J., Teo, C.-P., and Qian, L. (2006): "Many-to-One Stable Matching: Geometry and Fairness." Mathematics of Operations Research, 31: 581-596.

Smith, A. (1796): An Inquiry into the Nature and Causes of the Wealth of Nations. Oxford University Press, Oxford.

Tan, J. (1991): "A Necessary and Sufficient Condition for the Existence of a Complete Stable Matching." Journal of Algorithms, 12: 154-178.

Teo, C.-P. and Sethuraman, J. (1998): "The Geometry of Fractional Stable Matchings and its Applications." Mathematics of Operations Research, 23: 874-891. 